\title{
Defeito Total do Septo Atrioventricular. Correlação Anatomofuncional entre Pacientes com e sem Síndrome de Down
}

\author{
Liliana María Ferrín, Edmar A tik, Nana Miura Ikari, Tâmara Cortez Martins, \\ Miguel Barbero Marcial, Munir Ebaid
}

São Paulo, SP

\begin{abstract}
Objetivo - Comparar o grau de repercussão, fatores responsáveis e época de aparecimento dos sintomas do defeito do septo atrioventricular (DSAV), em pacientes com e sem síndrome de Down.

Métodos - Foram estudados 80 pacientes com idade $<2$ anos, sendo 55 (69\%) com síndrome de Down-grupo $I(G I)$ e $25(31 \%)$ sem síndrome de Down - grupo II (GII). Avaliaram-se a idade de manifestação dos sintomas, sua intensidade, classe funcional (CF), repercussão clínica, tipo anatômico e grau de malformação da valva atrioventricular (VAV).

Resultados - A idade média de manifestação dos sintomas foi de 50( \pm 75$)$ dias nos dois grupos. A CF II (NYHA) predominou no GI (31 casos - 56,5\%) e a CF III-IV no GII (19-76\%) $p<0,005$. O quadro de insuficiência cardíaca esteve presente em 34 (62\%) pacientes do GI e em 21 (84\%) do GII e, o de hipertensão pulmonar em 21 (38\%) do GI e em 4 (16\%) do GII, $p<0,04$. Pressão média da artéria pulmonar $>50 \mathrm{mmHg}$ ocorreu em $56 \%$ dos casos do GI em relação a $28 \%$ do GII p <0,019. A evolução até a cirurgia foi instável (agravamentos da insuficiência cardíaca) em 33 (60\%) do GI e em 21 (84\%) do GII p<0,03. O tipo A de Rastelli foi o mais encontrado nos dois grupos, 35 (67\%) pacientes no GI e nos 25 (100\%) do GII. A alteração anatômica da VAV foi importante em $8 \%$ no GI e em $38 \%$ no GII.

Conclusão - Há sugestão de predominância de hiperreatividade vascular pulmonar nas crianças com síndrome de Down e de manifestações de insuficiência cardíaca nas geneticamente normais.
\end{abstract}

Palavras-chave: defeito do septo atrioventricular, síndrome de Down, hipertensão pulmonar

\section{Complete Atrioventricular Canal. Clinical- Anatomic Correlations between Patients with and without Down's Syndrome}

Purpose - To compare clinical course, causes and symptoms beginning enset time in children with complete atrioventricular canal with and without Down's syndrome.

Methods - Records of 80 patients <2 years of age, were reviewed. There were 55 (69\%) with Down's syndrome-group I(GI) and 25 (31\%) without - group II(GII). Age at synpton enset intensity, functional class, clinical repercussion and anatomic variations in patients undergoing corrective surgery were evaluated.

Results - Mean age at symptoms onset was similarfor the two groups $(50 \pm 75$ days). Class II (NYHA) was more frequent in GI (31 patients - 56.5\%) and class III-IV (NYHA) in GII (19 patients - 76\%) p<0.005. Clinical repercussion evaluation showed that congestive heart failure was present in 34 (62\%) patients of GI and 21 (84\%) of GII; and, pulmonary hypertension was in 21 (38\%) patients of GI and $4(16 \%)$ patients of GII $p<0.04$. Mean pulmonary arterial pressure of $50 \mathrm{mmHg}$ or more was present in $68 \%$ of children with Down's syndrome and in $35 \%$ of GII. Clinical course until surgical correction was down hill in 33 (60\%) from GI and 21 (84\%) from GII p<0.03. Seventy seven patients underwent surgical correction. CAVC type A of the Rastelli classification was predominant in both groups, GI 37 (67\%) - GII 25 (100\%). There or more severe valvar morphologic lesions in group II $(38 \%)$ than in group I (8\%).

Conclusion - There seems to be a pulmonary vascular hyperreactivity predominance in Down's children and cardiac insufficiency signs in the normal genetic group.

Key-words: complete atrioventricular canal, Down's syndrome, pulmonary hypertension.

Arq Bras Cardiol, volume 69 (n' 1), 19-23, 1997

Instituto do Coração do Hospital das Clínicas - FMUSP

Correspondência: Incor - Liliana María Ferrín - Incor - Cardiologia Pediátrica - Av.

Dr. Enéas C. Aguiar, 44 - 05403-000 - São Paulo - SP

Recebido para publicação em 9/1/97

Aceito em 6/5/97
O defeito do septo atrioventricular (DSAV) é uma malformação cardíaca relativamente freqüente, cuja prevalência é, em geral, cerca de 3\% das cardiopatias congênitas. Está associado geralmente aos portadores da síndrome de Down, tornando-se o defeito mais representativo ${ }^{1-3}$. Seu 
quadro clínico, em decorrência das alterações anatomofuncionais, caracteriza-se basicamente por hiperfluxo pulmonar, de manifestação precoce, nas primeiras semanas de vida. A complicação pulmonar tipo hiper-resistência com posterior doença vascular oclusiva tem uma expressão maior nessas crianças nos meses subseqüentes, ao contrário dos não portadores desta síndrome ${ }^{4-6}$.

O objetivo deste trabalho é avaliar não apenas a repercussão hemodinâmica, como a época de aparecimento dos sintomas, comparando os achados nos dois grupos de pacientes e estabelecer prognóstico, conduta e idade mais adequada para a correção operatória, além de avaliar eventuais fatores relacionados às principais diferenças nesses pacientes.

\section{Métodos}

Foram estudados, retrospectivamente, 80 pacientes, acompanhados no INCOR, de janeiro/87 a maio/95, portadores de DSAV apenas na forma total e com idades até dois anos, tendo sido excluídos os com qualquer outro defeito associado, exceto o ductus arteriosus persistente e divididos em dois grupos, segundo a presença ou ausência da síndrome genética. Avaliaram-se a idade de apresentação dos sintomas, assim como sua intensidade, a classe funcional, evolução clínica após a $1^{\mathrm{a}}$ consulta e o momento da correção cirúrgica. Considerou-se como evolução estável, quando o quadro clínico não sofria modificações e, evolução instável, quando o agravamento da insuficiência cardíaca era expressivo.

Ao eletrocardiograma (ECG), observaram-se, além do ritmo, a presença de bloqueio atrioventricular, sobrecarga de câmaras cardíacas e do bloqueio divisional ântero-superior esquerdo e, na radiografia de tórax, o aumento da área cardíaca e o aspecto da trama vascular pulmonar.

No ecocardiograma, além do tipo anatômico segundo a classificação de Rastelli (tipo A, B ou C), avaliaram-se a presença e grau de insuficiência da valva atrioventricular (VAV), e a hipertensão pulmonar (HP) pela determinação da pressão média da artéria pulmonar (PMAP).

No ato operatório foram verificados o tipo anatômico (Rastelli), a presença e severidade da malformação da VAV e, o tamanho das comunicações interatrial (CIA) e interventricular (CIV). A correção operatória baseou-se na técnica habitual de fechamento da CIA e CIV com reparos de pericárdio e a plastia da VAV.
Para o tratamento estatístico aplicaram-se a análise descritiva e a comparação de grupos, sendo utilizados o teste exato de Fisher (2-tail), o teste T de Student e o $x^{2}$ (quiquadrado) quando apropriado.

\section{Resultados}

Entre os 80 pacientes estudados, 55 (69\%) eram portadores da síndrome de Down constituindo o grupo I (GI) e 25 (31\%) sem síndrome genética, o grupo II (GII). Eram do sexo feminino, $51(63,7 \%)$ pacientes, 34(62\%) no GIe 17 (68\%) no GII e do sexo masculino, $29(36,2 \%)$ pacientes, $21(38,2 \%)$ no GIe 8(32\%) no GII pNS.

A idade de manifestação dos sintomas foi de 1 a 390 dias para o GI (média 49 dias \pm 77 ); e de 1 a 270 dias para o GII (média 52 dias \pm 73 ) pNS. A dispnéia foi o sintoma principal de apresentação e variou em intensidade, determinando diferença estatisticamente significante em relação à $\mathrm{CF}$ (NYHA) nos dois grupos, com predomínio da CF II no grupo I e da CF III-IV no grupo II - p:0,001 (tab. I).

$\mathrm{Na}$ avaliação dos exames complementares, o ECG mostrou ritmo sinusal em todos os pacientes com predomínio dos sinais de sobrecarga biventricular (SBV) sobre a sobrecarga ventricular direita (SVD) isolada nos dois grupos, 34 (62\%) casos no GI e 16(64\%) no GII (pNS). OBDAS esteve presente em todas as crianças dos dois grupos com exceção de quatro (7\%) pertencentes ao GI (pNS). A radiografia de tórax mostrou uma tendência ao aumento importante da área cardíaca no grupo II (10 pacientes - 40\%) e, ao moderado no grupo I (31 pacientes - 57\%), sendo a trama vascular pulmonar aumentada na maioria dos casos em ambos os grupos pNS (tab. II).

O tipo anatômico, segundo a classificação de Rastelli, avaliado pelo ecocardiograma e confirmado no intra-operatório, foi para o GI, 37 (67\%) pacientes do tipo A, cinco (9\%) do tipo B e 13 (24\%) do tipo C; já no GII todos os 25 pacientes eram do tipo A $(100 \%)(\mathrm{p}<0,005)$. A avaliação ecocardiográfica da disfunção da VAV, demonstrou uma distribuição semelhante do grau de insuficiência nos dois grupos, predominando a moderada no GI:13 (24\%) pacientes e no GII - sete $(29 \%)$ (pNS). A PMAP, avaliada pelo ecocardiograma Doppler, variou de 20 a $67 \mathrm{mmHg}$ (média 49,3 $\mathrm{mmHg}$ \pm 10 ) no GI e no GII de 30 a 70mmHg (média 44,6mmHg \pm 9). Foi $>50 \mathrm{mmHg}$ em $31(56 \%)$ pacientes do GI e em sete (28\%) do GII $(\mathrm{p}<0,019)$ (tab. II).

\begin{tabular}{|c|c|c|c|c|c|}
\hline \multirow[t]{2}{*}{$\begin{array}{l}\text { Idade de manifestação } \\
\text { dos sintomas }\end{array}$} & \multicolumn{2}{|c|}{$\begin{array}{c}\text { GRUPO I } \\
1 \text { dia - } 390 \text { dias } \\
\text { média } 49 \text { dias }\end{array}$} & \multicolumn{2}{|c|}{$\begin{array}{c}\text { GRUPO II } \\
1 \text { dia - } 270 \text { dias } \\
\text { média } 52 \text { dias }\end{array}$} & \multirow[t]{2}{*}{$\begin{array}{c}\mathrm{p} \\
0.88\end{array}$} \\
\hline & $\mathrm{n}$ & $\%$ & $\mathrm{n}$ & $\%$ & \\
\hline CF I & 3 & 5,5 & 1 & 4 & \\
\hline CF II & 31 & 56,5 & 5 & 20 & 0,005 \\
\hline CF III - IV & 21 & 38 & 19 & 76 & \\
\hline
\end{tabular}




\begin{tabular}{|c|c|c|c|c|c|c|}
\hline \multicolumn{7}{|c|}{ Tabela II - Elementos de exames complementares } \\
\hline \multirow{2}{*}{\multicolumn{2}{|c|}{ ECG }} & \multicolumn{2}{|c|}{ GRUPO I } & \multicolumn{2}{|c|}{ GRUPO II } & \multirow[b]{2}{*}{$\mathrm{p}$} \\
\hline & & $\mathrm{n}$ & $\%$ & $\mathrm{n}$ & $\%$ & \\
\hline \multicolumn{2}{|c|}{ SBV } & 34 & 62 & 16 & 64 & \\
\hline \multirow{2}{*}{\multicolumn{2}{|c|}{$\begin{array}{l}\text { SVD } \\
\text { BDASE }\end{array}$}} & 21 & 38 & 9 & 36 & NS \\
\hline & & 51 & 93 & 25 & 100 & NS \\
\hline \multicolumn{7}{|c|}{ Radiografia de tórax } \\
\hline \multirow{4}{*}{$\mathrm{AC}$} & Normal & 1 & 2 & - & - & \multirow{4}{*}{ NS } \\
\hline & Discreta & 11 & 20 & 7 & 28 & \\
\hline & Moderada & 31 & 57 & 8 & 32 & \\
\hline & Importante & 11 & 20 & 10 & 40 & \\
\hline \multirow{3}{*}{ TVP } & Normal & 10 & 18 & 3 & 12 & \multirow{3}{*}{ NS } \\
\hline & Aumentada & 34 & 61 & 18 & 72 & \\
\hline & Diminuída & 11 & 20 & 4 & 16 & \\
\hline \multicolumn{2}{|c|}{ Ecocardiograma } & \multicolumn{2}{|c|}{ GRUPO I } & \multicolumn{2}{|c|}{ GRUPO II } & \\
\hline \multicolumn{2}{|c|}{$\begin{array}{l}\text { Tipo anatômico } \\
\text { (Rastelli) }\end{array}$} & $\mathrm{n}$ & $\%$ & $\mathrm{n}$ & $\%$ & $\mathrm{p}$ \\
\hline & A & 37 & 67 & 25 & 100 & \multirow{3}{*}{0.005} \\
\hline & B & 5 & 9 & - & - & \\
\hline & $\mathrm{C}$ & 13 & 24 & - & - & \\
\hline \multirow[t]{6}{*}{ Insuficiê } & & & & & & \\
\hline & VAV & $\mathrm{n}$ & $\%$ & $\mathrm{n}$ & $\%$ & $\mathrm{p}$ \\
\hline & Normal & 26 & 48 & 7 & 29 & \multirow{4}{*}{ NS } \\
\hline & Discreta & 11 & 20 & 7 & 29 & \\
\hline & Moderada & 13 & 24 & 7 & 29 & \\
\hline & Importante & 4 & 8 & 3 & 13 & \\
\hline \multicolumn{2}{|l|}{ PMAP } & \multicolumn{2}{|c|}{$20-67 \mathrm{mmHg}$} & \multicolumn{2}{|c|}{$30-70 \mathrm{mmHg}$} & \\
\hline \multicolumn{2}{|l|}{ (média) } & \multicolumn{2}{|c|}{$49.3 \mathrm{mmHg}$} & \multicolumn{2}{|c|}{$44.6 \mathrm{mmHg}$} & NS \\
\hline \multicolumn{2}{|c|}{$>50 \mathrm{mmHg}$} & $31 \mathrm{pts}$ & $56 \%$ & $7 \mathrm{pts}$ & $28 \%$ & 0.019 \\
\hline $\begin{array}{l}\text { ECG- el } \\
\text { cardíaca }\end{array}$ & $\begin{array}{l}\text { iograma; } \mathrm{SE} \\
\text { rama vascul }\end{array}$ & $\begin{array}{l}\text { iventric } \\
\text { V- valv }\end{array}$ & $\begin{array}{l}\text { sobrec } \\
\text { ular; }\end{array}$ & $\begin{array}{l}\text { DASE } \\
\text { artéria }\end{array}$ & ivision & uerdo; AC- áre \\
\hline
\end{tabular}

O cateterismo cardíaco foi realizado em 31 pacientes, sendo os dados hemodinâmicos semelhantes aos achados ecocardiográficos no referente à PMAP.

O quadro clínico de insuficiência cardíaca (ICC), caracterizado por dispnéia moderada a intensa, hepatomegalia maior que $3 \mathrm{~cm}$ da reborda costal direita, $\mathrm{SBV}$ predominante e área cardíaca e trama vascular pulmonar moderada a intensamente aumentada na radiografia de tórax, ocorreu em 34 $(61,8 \%)$ pacientes no GI e em 21 (84\%) no GII. O quadro clínico sugestivo de HP, caracterizado por discreta dispnéia, hepatomegalia até $2 \mathrm{~cm}$ da reborda costal direita, SVD predominante ao ECG, área cardíaca discreta a moderadamente aumentada e trama vascular pulmonar diminuída na periferia na radiografia de tórax, foi encontrado em $21(38,2 \%)$ pacientes no GI e em quatro $(16 \%)$ no GII ( $\mathrm{p}<0,04)$. A evolução clínica, seja da ICC ou HP, avaliada desde o início do acompanhamento até a cirurgia (mínimo 27 dias - máximo 1079 dias), foi estável em 22 (40\%) pacientes do GI, em relação a quatro (16\%) do GII. Piora evolutiva do quadro clínico, apesar do uso de medicação anticongestiva e/ou vasodilatadora pul- monar, ocorreu em $33(60 \%)$ pacientes do GI e em 21 (84\%) do GII $(\mathrm{p}<0,03)$ (tab. III).

Dos 80 pacientes, três não foram submetidos a tratamento cirúrgico. Um, pertencente ao GI, por decisão dos pais em decorrência da síndrome genética, com evolução estável no seguimento e dois ao GII: um apresentando hiper-resistência pulmonar na ocasião da indicação da correção e outro acometido de acidente vascular cerebral hipóxico de desconhecida evolução posterior.

As idades por ocasião da cirurgia variaram de 30 a 720 (média de 247士159) dias no GI e de 90 a 1080 (média de 397 \pm 276$)$ dias no GII (p: 0,01).

Os achados cirúrgicos confirmaram o tipo anatômico da malformação descrita ao ecocardiograma. Observaramse alterações da VAV, sendo consideradas importantes: displasia com fibrose, redundância, duplo aparelho mitral, retração das cúspides, além de anomalias no aparelho subvalvar: músculo papilar único, retração das cordas em quatro (8\%) pacientes do GI e em oito (38\%) do GII; como moderada: displasia das cúspides em sete (14\%) do GI e em 


\begin{tabular}{|c|c|c|c|c|c|}
\hline \multicolumn{6}{|c|}{ Tabela III - Aspectos funcionais e evolutivos } \\
\hline & \multicolumn{2}{|c|}{ GRUPO I } & \multicolumn{2}{|c|}{ GRUPO II } & \multirow{2}{*}{$\mathrm{p}$} \\
\hline & $\mathrm{n}$ & $\%$ & $\mathrm{n}$ & $\%$ & \\
\hline $\begin{array}{l}\text { ICC(dispnéia moderada } \\
\text { a intensa, hepatomegalia }>3 \mathrm{~cm} \text {, } \\
\text { SBV, AC e TVP aum) }\end{array}$ & 34 & 61,8 & 21 & 84 & 0,04 \\
\hline $\begin{array}{l}\text { HP (dispnéia discr., hepatomeg. } \\
\text { até } 2 \mathrm{~cm} \text {, AC aum.disc.a mod, } \\
\text { TVP norm. ou dim.) }\end{array}$ & 21 & 38,2 & 4 & 16 & \\
\hline \multirow[t]{2}{*}{ Evolução } & \multicolumn{2}{|c|}{ GRUPO I } & \multicolumn{2}{|c|}{ GRUPO II } & $\mathrm{p}$ \\
\hline & $\mathrm{n}$ & $\%$ & $\mathrm{n}$ & $\%$ & \\
\hline Período & \multicolumn{2}{|c|}{$\begin{array}{c}7-713 \text { dias } \\
(\mathrm{m}=204 \text { dias })\end{array}$} & \multicolumn{2}{|c|}{$\begin{array}{r}90-1079 \text { dias } \\
(\mathrm{m}=345 \text { dias })\end{array}$} & \\
\hline Estável & 22 & 40 & 4 & 16 & 0,03 \\
\hline Instável & 33 & 60 & 21 & 84 & \\
\hline
\end{tabular}

4(19\%) do GII; como discreta: fibrose, retração ou redundância de alguma cúspide em nove (18\%) do GI, sem nenhum paciente do GII. A valva foi considerada sem estes tipos de alterações em 27 (54\%) pacientes do GI e em oito (38\%) do GII $\mathrm{p}<0,009$ (tab. IV). Nenhuma diferença foi observada entre os grupos no que se refere ao tamanho das CIA e CIV.

No período pós-operatório imediato faleceram nove (16\%) pacientes, todos do GI, sete por persistência de pressão elevada na artéria pulmonar e dois por complicação infecciosa na $2^{\mathrm{a}}$ semana pós-cirurgia.

\section{Discussão}

A história natural dos pacientes com DSAV demonstra uma sobrevida de $54 \%$ aos seis meses de idade e de $15 \%$ aos dois anos. A mortalidade no lactente está relacionada a
ICC e, na infância, ocorre em decorrência da doença vascular pulmonar oclusiva ${ }^{4,5,8,9}$.

Vários autores ${ }^{4,5,7}$ têm sugerido que crianças com síndrome de Down desenvolvem mudanças vasculares pulmonares mais precoce e rapidamente progressivas, que crianças não portadoras da síndrome, embora este último grupo se apresente com mais sintomas.

Na nossa série de 80 pacientes, a idade do início dos sintomas e sinais foi semelhante em ambos os grupos, sendo os sintomas principais a dispnéia de esforço e as broncopneumonias de repetição. A intensidade desses sintomas, avaliada segundo a CF (NYHA), mostrou diferenças estatisticamente significativas, com maior repercussão clínica nos pacientes sem a síndrome de Down (CF III-IV) que naqueles portadores da mesma $(\mathrm{CF} I I)(\mathrm{p}<0,005)$. A diferença foi também significativa em relação à evolução pré-operató-

\begin{tabular}{|c|c|c|c|c|c|c|}
\hline \multicolumn{7}{|c|}{ Tabela IV - Achados cirúrgicos } \\
\hline & & \multicolumn{2}{|c|}{ GRUPO I } & \multicolumn{2}{|c|}{ GRUPO II } & \multirow[b]{2}{*}{$\mathrm{p}$} \\
\hline & & $\mathrm{n}$ & $\%$ & $\mathrm{n}$ & $\%$ & \\
\hline \multirow[t]{2}{*}{ Pacientes operados } & & 54 & 98 & 23 & 92 & NS \\
\hline & $\mathrm{N}$ & 27 & 54 & 8 & 38 & \\
\hline Malformação & $\mathrm{D}$ & 9 & 18 & - & - & \\
\hline \multirow[t]{2}{*}{ VAV } & M & 7 & 14 & 4 & 19 & 0.009 \\
\hline & I & 4 & 8 & 8 & 38 & \\
\hline \multirow[t]{2}{*}{ CIA } & $\mathrm{P}$ & 21 & 42 & 7 & 28 & \\
\hline & $\mathrm{G}$ & 29 & 58 & 18 & 72 & NS \\
\hline \multirow[t]{2}{*}{ CIV } & $\mathrm{P}$ & 14 & 29 & 7 & 28 & \\
\hline & $\mathrm{G}$ & 35 & 71 & 18 & 72 & NS \\
\hline Óbitos & & 9 & 16 & - & - & 0,02 \\
\hline
\end{tabular}


ria, tendo apresentado maior instabilidade as crianças geneticamente normais, apesar do tratamento médico adequado $(\mathrm{p}<0,03)$. Na correlação clínica das variáveis estudadas, em relação aos aspectos predominantes em cada grupo, o quadro de HP foi mais freqüente, quando presente a síndrome de Down. O quadro de ICC predominou nos casos sem síndrome de Down, sendo esta diferença estatisticamente significante $(\mathrm{p}<0,04)$, apesar de não ter sido possível a análise multivariável.

Newfeld e $\mathrm{col}^{4}$ demonstraram que a PMAP $>50 \mathrm{mmHg}$ tinha boa correlação com a classificação histológica de Heath - Edwards em grau III-IV nas crianças $>2$ anos. Esta correlação não foi possível em lactentes, provavelmente em decorrência do maior componente de espasmo na musculatura vascular pulmonar nessa faixa etária. Na nossa série, não encontramos diferença significativa nos valores de PMAP entre pacientes com e sem síndrome de Down, quando estudada como variável única, com média semelhante para ambos os grupos. No entanto, quando foi avaliada presença de PMAP > 50mmHg, esta foi mais encontrada no GI, correlacionando-se com o quadro clínico de HP(GI: 31 pacientes - 56\%; GII:7 pacientes - 28\%)

Haworth e col ${ }^{10}$ observaram dois padrões clínicos de reatividade vascular pulmonar na presença de grandes shunts intracardíacos. No $1^{\circ}$ padrão, a regressão pós-natal normal da musculatura arteriolar pulmonar permite o aparecimento de shunt da esquerda à direita e insuficiência cardíaca de graus variáveis. No $2^{\circ}$ padrão, menos comum, a musculatura arteriolar pulmonar não regride normalmente após o nascimento, permanecendo resistência vascular pulmonar elevada que impede o aumento importante do fluxo sangüíneo pulmonar. Este padrão corresponderia aos pacientes com síndrome de Down e resistência vascular pulmonar elevada fixa no $1^{\circ}$ ano de vida, de acordo com o observado por outros autores e no nosso material.

Dentre os aspectos morfológicos, é importante notar a predominância do tipo A de Rastelli nos dois grupos, enquanto que os tipos $\mathrm{B}$ e $\mathrm{C}$ foram encontrados exclusivamente em pacientes com a síndrome genética $(\mathrm{p}<0,005)$, relatado também em outras séries ${ }^{2,6,8,9}$.

A magnitude da malformação da VAV esteve relacionada ao quadro clínico, sendo maior nos pacientes com cromossomas normais ( $p<0,009$, ). Não houve diferença significante no grau de insuficiência valvar que possa explicar maior instabilidade hemodinâmica neste grupo, como fora salientado por outros autores ${ }^{5,6,11-14}$. É possível que a maior alteração da VAV, associada às malformações do aparelho subvalvar mitral (músculo papilar único, cordas redundantes, etc) determinem, ao lado da menor reatividade vascular pulmonar observada, pior repercussão clínica naqueles sem a anomalia genética. No entanto, estudos prospectivos e específicos são necessários para esclarecer estes aspectos.

As diferenças clínicas, estruturais e evolutivas significativas achadas entre os dois grupos, com as devidas considerações decorrentes do fato de ser um estudo retrospectivo, orientam para: sugestão de predominância da hiperreatividade vascular pulmonar nos pacientes com síndrome de Down, manifestação mais intensa e evolução pior com instabilidade hemodinâmica e alterações anatômicas mais acentuadas nas crianças sem a síndrome, o que ocasiona a indicação operatória mais precoce neste grupo.

\section{Referências}

1. Fixler DE, Pastor P, Chamberlin M, Sigman E, Eifler CW -Trends in congenital heart disease in Dallas country - births 1971-1984. Circulation 1986; 81: 137-42.

2. Geva T, Ayres NA, Pignatelli RH, Gajarski RJ - Echocardiographic evaluation of common atrioventricular canal defects: A study of 206 consecutive patients. Echocardiography 1996; 13: 387-99.

3. Baekgaard Laursen H - Congenital heart disease in Down's syndrome. Br Heart J 1976; 38: 32-8.

4. Newfeld E, Sher M, Paul MH, Nikaidoh H - Pulmonary vascular disease in complete atrioventricular canal defects. Am J Cardiol 1977; 39: 721-6.

5. Clapp S, Perry BL, FarookiZQ et al - Down's syndrome, common atrioventricular canal, and pulmonary vascular obstructive disease. J Thorac Cardiovasc Surg 1990; 100: 115-21.

6. Mc Cabe J, Engle MA, Gay W, EbertP-Surgical treatment of endocardial cushion defects. Am J Cardiol 1977; 39: 72-7.

7. Shaher R, Farina M, Porter I, Bishop M - Clinical aspects of congenital heart disease in mongolism. Am J Cardiol 1972; 29: 497-503.

8. Clapp S, Perry BL, Farooki ZQ et al - Surgical and medical results of complete atrioventricular canal: a ten year review. Am J Cardiol 1987; 59: 454-8.
9. Hanly F, Fenton K, Castañeda A et al - Surgical repair of complete atrioventricular canal disease in infancy. Twenty- year trends. J Thorac Cardiovasc Surgery 1993; 106: 387-97.

10. Haworth SG, Sauer U, Buhlmeyer K, Reid L-Development of the pulmonary circulation in ventricular septal defect: a quantitative structural study. Am J Cardiol 1977; 40: 781-8.

11. García Hernandez JA, Cáceres Espejo J, Soult Rubio JA et al - Evaluación de la corrección quirúrgica en los canales atrioventriculares completos. Rev Esp Cardiol 1993; 46: 293-7.

12. Bonnets P, Goldberg S, Copeland J-Frequency of left atrioventricular valve regurgitation after repair of complete atrioventricular defect. Am J Cardiol 1994; 74: 1157-60.

13. Haworth SG - Pulmonary vascular bed in children with complete atrioventricular septal defect: relation betwen structural and hemodynamic abnormalities. Am J Cardiol 1986; 57: 833-9.

14. Rabinovitch M, Haworth SG, Castañeda A, Nadas AS, Reid LM - Lung biopsy in congenital heart disease: a morphometric approach to pulmonary vascular disease. Circulation 1978; 58: 1107-22. 